\title{
Evaluating the non-tuberculous mycobacteria effect in the tuberculosis infection diagnosis
}

\author{
I. Latorre*,\#,ף, M. De Souza-Galvão\#,+, J. Ruiz-Manzano\#,ף,§, A. Lacoma*,\#,ף,

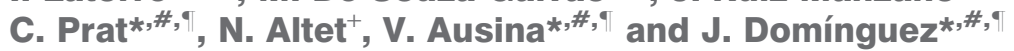

ABSTRACT: The aim of the present study was to determine the role of previous non-tuberculous mycobacteria sensitisation in children as a factor of discordant results between tuberculin skin test (TST) and an in vitro T-cell based assay (T-SPOT.TB; Oxford Immunotec, Oxford, UK).

We enrolled 21 non-bacille Calmette-Guérin-vaccinated paediatric patients for suspicious of latent tuberculosis infection (LTBI). These patients yielded a positive TST and a negative T-SPOT.TB. Cells were stimulated with Mycobacterium avium sensitin (having cross-reaction with Mycobacterium intracellulare and Mycobacterium scrofulaceum) and the presence of reactive T-cells was determined by an ex vivo ELISPOT.

From the 21 patients, in 10 cases (47.6\%), we obtained a positive ELISPOT result after stimulation with $M$. avium sensitin, in six $(28.6 \%)$ cases, the result was negative and in the remaining five $(23.8 \%)$ cases, the result was indeterminate.

In conclusion, previous non-tuberculous mycobacteria sensitisation induces false-positive results in the TST for diagnosing LTBI and the use of $\gamma$-interferon tests could avoid unnecessary chemoprophylaxis treatment among a child population.

KEYWORDS: Childhood, ELISPOT, interferon- $\gamma$ release assays, latent tuberculosis infection, Mycobacterium avium sensitin, non-tuberculous mycobacteria

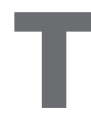
he detection and treatment of active TB is a key strategy in the control of childhood tuberculosis (TB) [1]. Children have a high risk of progression to active TB [2]. Therefore, a rapid and specific diagnosis of latent $\mathrm{TB}$ infection (LTBI) is essential in preventing the progression to disease. The tuberculin skin test (TST) attempts to measure cell-mediated immunity in the form of a delayed-type hypersensitivity response to the purified protein derivative (PPD) [3]. The biggest drawback of TST is that individuals sensitised by previous exposure to non-tuberculous mycobacteria (NTM) or vaccinated with Mycobacterium bovis bacilli Calmette-Guérin (BCG) respond immunologically to PPD. Consequently, unnecessary latent tuberculosis treatments are prescribed.

In vitro assays for measuring T-cell-mediated immune responses have been developed. In these assays, infected individuals are identified by the detection of $\gamma$-interferon (IFN- $\gamma$ ) released by the T-cells that are sensitised after being stimulated with the specific Mycobacterium tuberculosis (MTB) antigens of region of deletion (RD) 1 (early-secreted antigenic target protein (ESAT)-6 and $10-\mathrm{kD}$ culture filtrate protein (CFP)-10) $[4,5]$. Promising results from these diagnostic tests in both adults and children have been published [6-11].

However, there are several discordant results between the IFN- $\gamma$ tests and the TST [12]. One of the more challenging correct interpretations remains in the instance of positive TST and negative IFN- $\gamma$ results in non-BCG vaccinated children. In our experience [7], among unvaccinated children with a positive TST, the T-SPOT.TB result was negative in $56.6 \%$ of the cases.

The aim of the present study was to determine the role of previous NTM sensitisation in children as a factor of discordant results between TST and the T-SPOT.TB test.

\section{MATERIAL AND METHODS}

\section{Patients and inclusion criteria}

We retrospectively enrolled a total of 21 paediatric patients, who attended Hospital Universitari Germans Trias i Pujol (Badalona, Spain) or TB Control and Prevention Unit of Barcelona (CAP

\section{AFFILIATIONS}

*Servei de Microbiologia,

sServei de Pneumologia, Hospital Universitari "Germans Trias i Pujol", Fundació Institut d'Investigació en Ciències de la Salut Germans Trias i Pujol,

- Ciber Enfermedades Respiratorias, Instituto de Salud Carlos III, Badalona,

\#Universitat Autònoma de Barcelona. Bellaterra, and

+Unidad de Prevención y Control de la Tuberculosis de Barcelona,

Barcelona, Spain.

\section{CORRESPONDENCE}

J. Domínguez

Servei de Microbiologia

Fundació Institut d'Investigació en

Ciències de la Salut "Germans Trias i

Pujol"

Carretera del Canyet s/n

08916 Badalona

Barcelona

Spain

E-mail: jadomb@gmail.com

Received:

Dec 282008

Accepted after revision:

April 072009 
Drassanes, Barcalona, Spain) for suspicion of LTBI. These patients were enrolled for contact tracing studies or for screening of LTBI. Inclusion criteria for this selected population were a positive TST, a negative T-SPOT.TB, non-BCG vaccination and no more than 2 weeks of chemoprophylaxis when blood sampling. None of the children presented lymphadenitis at the time of inclusion. We have also included control groups to validate the methodology and the results: 11 children with both TST and T-SPOT.TB negative results, and six individuals with microbiologically confirmed $M$. avium infection (four lymphadenitis and two respiratory infections). Another additional group of 10 children with both TST- and TSPOT.TB-positive results was included in order to know the background of $M$. tuberculosis and $M$. avium sensitisation in the population. The main demographic characteristics of the groups included in the study are shown in table 1 .

Ethics approval for this study was provided by the corresponding Ethics Committees. We obtained written informed consent from all parents before blood sampling. A detailed questionnaire from all patients was completed to indicate the results of any previous TST, BCG vaccination status, details of any contact with a person diagnosed of active TB, history of prior active TB, LTBI and HIV infection, chest radiography and other medical conditions.

\section{TST}

Two intradermal tuberculin units of PPD RT23 Tween 80 (Statens Serum Institut, Copenhagen, Denmark) were used to perform TST. The tuberculin was administered using Mantoux method, and the size of the induration was interpreted after $48-72 \mathrm{~h}$ by trained personnel. In this study, TST indurations $\geqslant 5 \mathrm{~mm}$ were classified as positive [13].

\section{Detection of T-cell sensitised against MTB specific antigens}

Peripheral blood mononuclear cells (PBMCs) were stimulated with ESAT-6 and CFP-10 antigens individually. The presence of reactive antigen-specific $\mathrm{T}$-cells was revealed by ELISPOT (T-SPOT.TB; Oxford Immunotec, Oxford, UK). The test was performed in accordance with the manufacturer's instructions. Unstimulated cells were washed with RPMI medium (Invitrogen, Auckland, New Zealand) and resuspended in freeze medium (80\% RPMI and 20\% free bovine serum (PAA Laboratories $\mathrm{GmbH}$, Pasching, Austria)), adding dropwise $10 \%$ DMSO (Merck, Darmstadt, Germany) and frozen at $-80^{\circ} \mathrm{C}$.

TABLE 1 Demographic characteristics of patients studied

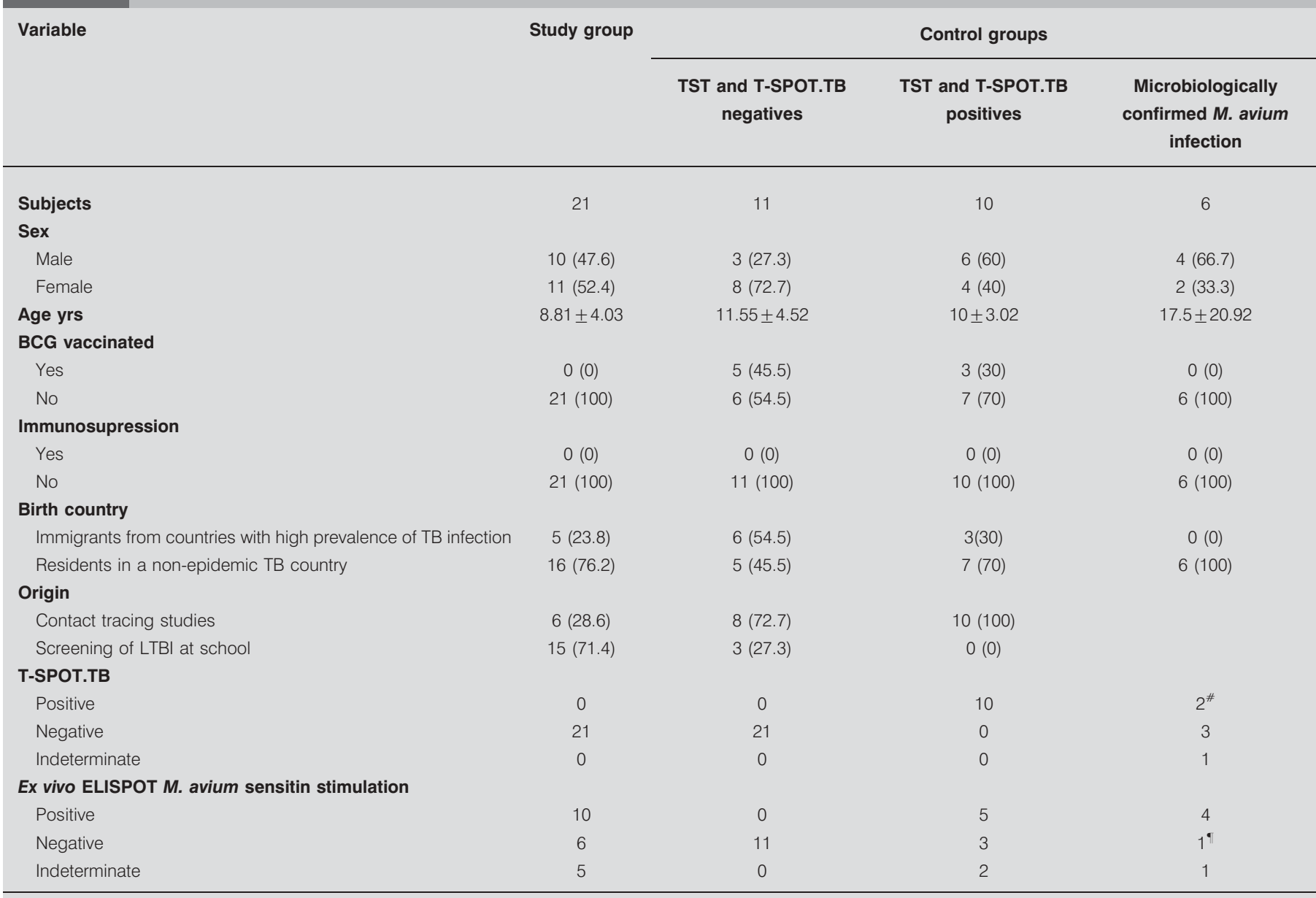

Data are presented as $\mathrm{n}, \mathrm{n}(\%)$ or mean $\pm \mathrm{SD}$. TST: tuberculin skin test; $M$. avium: Mycobacterium avium; BCG: bacilli Calmette-Guérin; TB: tuberculosis; LTBI: latent TB infection. ${ }^{*}$ : in one case, active TB was documented 8 yrs before; ${ }^{\top}: M$. avium infection was reported 2 yrs before. 


\section{Detection of T-cell sensitised against NTM sensitin}

The stimulation of the T-cells was performed using M. avium sensitin (Statens Serum Institute, Copenhagen, Denmark). The manufacturer informed that this sensitin has cross reaction with Mycobacterium intracellulare and Mycobacterium scrofulaceum. In order to perform ex vivo ELISPOT, stimulating with $M$. avium sensitin, cells were thawed and re-suspended in $10 \mathrm{~mL}$ of RPMI medium. Finally, cells were washed, re-suspended in AIM-V medium (Invitrogen, Auckland, New Zealand) and stimulated with medium alone (as nil control), phytohaemagglutinin (as positive control) and $M$. avium sensitin at a concentration of $10 \mu \mathrm{g} \cdot \mathrm{mL}^{-1}$. Plates were incubated for $16-20 \mathrm{~h}$ at $37^{\circ} \mathrm{C}$ with $5 \%$ $\mathrm{CO}_{2}$. Following incubation, wells were washed with PBS and incubated for $1 \mathrm{~h}$ at $2{ }^{\circ} \mathrm{C}$ with a monoclonal antibody to IFN- $\gamma$ conjugated to alkaline phosphatase. The presence of reactive antigen-specific T-cells was revealed as a spot in the well.

\section{Interpretation of the results}

Spots were scored using an automated ELISPOT plate reader (Lector AID Elispots; Autoimmun Diagnostiks $\mathrm{GmbH}$, Germany). All readings were also manually verified. The results of the assays were expressed as ESAT-6, CFP-10 and $M$. avium sensitin specific responder cells per million PBMCs. Test wells were scored as positive if the number of responder cells per million PBMCs minus their number in the control negative was $>24$. The result of the assay was considered indeterminate if the number of positive control cells per million PBMCs was $<80$, and the response to both of the antigen panels was negative.

\section{RESULTS}

From the 21 children with positive TST and negative TSPOT.TB, a positive ELISPOT result after stimulation with $M$. avium sensitin was obtained in $10(47.6 \%)$ cases. In six $(28.6 \%)$ cases the result was negative and in the remaining five $(23.8 \%)$ cases the result was indeterminate. The number of responder T-cells after M. avium sensitin stimulation was significantly higher than the number of responder T-cells after specific MTB antigens (ESAT-6 and CFP-10) stimulation: $p=0.001$ and $\mathrm{p}<0.001$, respectively.

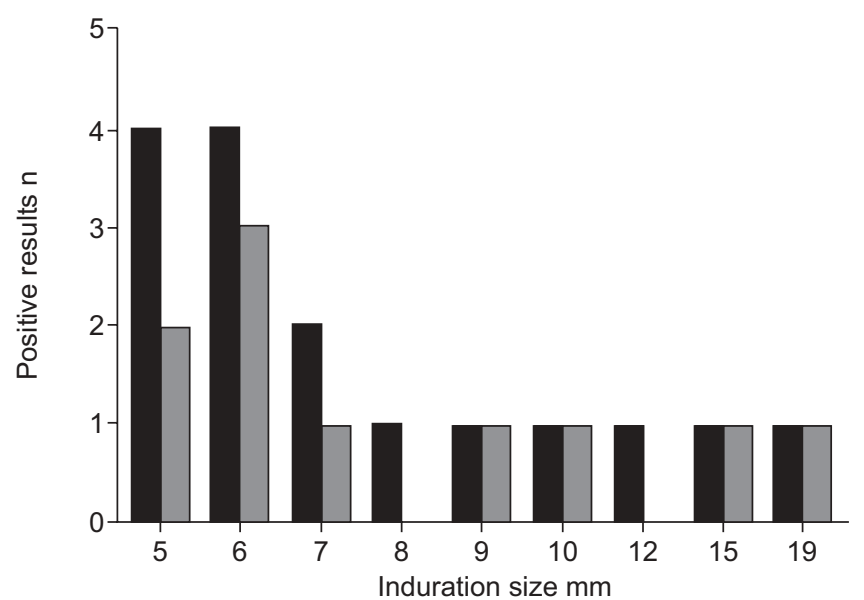

FIGURE 1. Induration size distribution of positive results of the tuberculin skin test (ם) and ex vivo Mycobacterium avium sensitin ELISPOT ( $\square$ ) among the children with a valid result.

Among the 10 children that obtained a positive result after stimulation with $M$. avium sensitin, five children were aged 6$7 \mathrm{yrs}$ and the other five children were aged 11-16 yrs. Additionally, eight were enrolled during LTBI screening at school and the remaining two, during a contact tracing study. Regarding the induration of the TST, eight of these children were in the range of $5-10 \mathrm{~mm}$, one case was $15 \mathrm{~mm}$ and the other case was $19 \mathrm{~mm}$ (fig. 1).

In all children with both TST- and T-SPOT.TB-negative results included as controls, negative ELISPOT results after stimulation with $M$. avium sensitin were obtained. There were no significant differences between the number of responder $\mathrm{T}$ cells after stimulation with ESAT-6, CFP-10 and M. avium sensitin. The differences in the number of responder T-cells to $M$. avium sensitin between the patients study group and this control group were significant $(p=0.004)$ (fig. 2). In the group of individuals with microbiologically confirmed $M$. avium infection, four out of five cases with valid results, cells

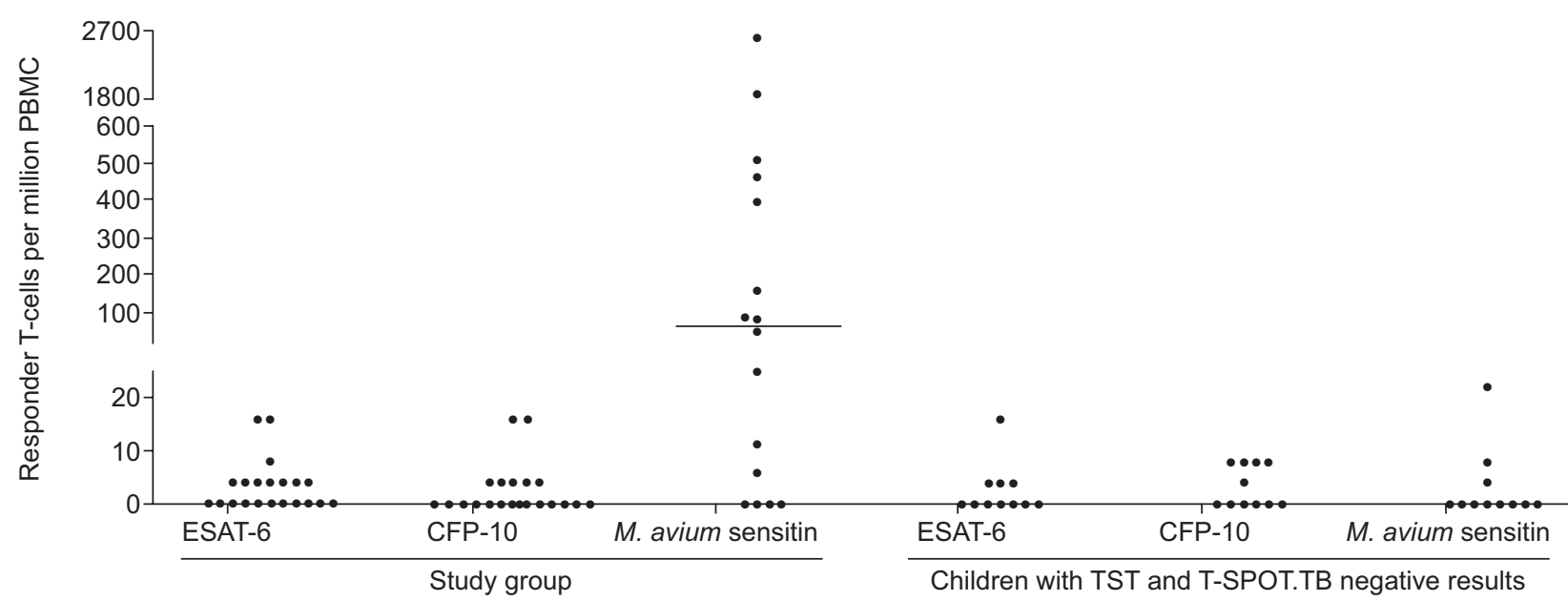

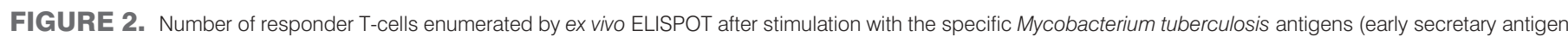

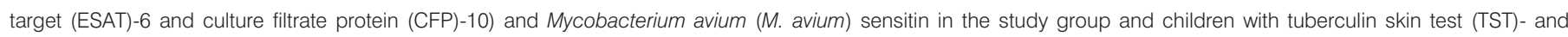
T-SPOT.TB-negative results. PBMC: peripheral blood mononuclear cells. 
sensitised against $M$. avium were detected. The results obtained by the study and all control groups are presented in table 1 . The indeterminate results were due to the low number of cells recovered after thawing.

\section{DISCUSSION}

Although specificity of IFN- $\gamma$ tests is excellent because the assay is not affected by BCG vaccination [6-8], frequent discordant results with TST have been described $[6,7,14]$. In fact, it has been recommended for priority research to obtain data to understand discordant TST and IFN- $\gamma$ tests results, including the role of NTM [12]. To date, the effect of NTM on IFN $-\gamma$ tests results has been poorly studied. In this sense, we have studied the effect of previous NTM sensitisation to try to give an explanation for the discordant results of positive TST and negative IFN- $\gamma$ results in non-BCG vaccinated children. Among the 16 children with a valid result, 10 (62.5\%) children had a specific response of T-cells after stimulation with $M$. avium sensitin.

It has been described that asymptomatic infections with $M$. avium and other NTM are common [15] and probably acquired in childhood [16-19]. In our area, the estimation of NTM infection in children with a positive TST $(5-10 \mathrm{~mm})$ ranged 20 $50 \%$ [16]. According with our results, using the ex vivo ELISPOT, eight $(80 \%)$ of the 10 children reactive against $M$. avium sensitin had a positive TST between 5 and $10 \mathrm{~mm}$, and nine $(90 \%)$ of them between 5 and $15 \mathrm{~mm}$. Indeed, in the children control group with TST- and T-SPOT.TB-positive results, the presence of T-cell sensitised against $M$. avium was detected in five out of the eight cases with valid result.

In our study, eight out of 10 children with a positive M. avium sensitin T-cell assay from our study group were enrolled from a routine screening of LTBI without known exposure to any active TB patient. Given that NTM infection affects the TST reading, it is in this group of children where IFN- $\gamma$ tests could be used to confirm the diagnosis in case of a positive TST result.

Regarding the six remaining discordant results without T-cell response after $M$. avium sensitin stimulation, there are three possible explanations. First, a real LTBI not detected by the IFN- $\gamma$ test. Nevertheless, the sensitivity of the IFN- $\gamma$ tests is considered to be higher than the TST, or at least at the same level. Secondly, the IFN- $\gamma$ test enumerates effectors T-cells that have recently been in contact with the antigen, in contrast, TST remains positive a long period after past $M$. tuberculosis infection [20]. However, in children the infection is usually recent. The third explanation is that the positive TST was due to a previous infection by a NTM without $M$. avium sensitin cross-reaction. It was impossible to test more NTM sensitins given that we didn't have more PBMCs stored from these patients.

One limitation of our study is that the skin test reactions to $M$. avium sensitin were not performed at the moment of inclusion of the children; therefore, it was not possible to correlate with the ex vivo result. Another limitation is that we have tested a reduced number of children. Nevertheless, despite these limitations, the results obtained are sufficiently consistent to draw some conclusions.
In summary, our results show enough evidence to state that previous NTM sensitisation in children induces false-positive results in the TST for diagnosing LTBI and that the IFN- $\gamma$ tests could avoid both unnecessary chemoprophylaxis treatment among child populations and consuming resources searching the index case.

\section{SUPPORT STATEMENT}

This work was supported by a grant from Sociedad Española de Neumología y Cirugía Torácica; Societat Catalana de Pneumologia (SOCAP); Fundació Catalana de Pneumologia (FUCAP); and Instituto de Salud Carlos III (RETIC RD06/0018). I. Latorre is a FPU pre-doctoral student and is the recipient of a grant from the Ministerio de Educación y Ciencia.

\section{STATEMENT OF INTEREST}

A statement of interest for J. Domínguez can be found at www.erj. ersjournals.com $/ \mathrm{misc} /$ statements.dtl

\section{ACKNOWLEDGEMENTS}

The authors would like to thank the nursing staff of the TB Control and Prevention Unit of Barcelona and C. Ramil, L. Haba, M. Ángel Cuesta, M. Pérez (all Servei de Microbiologia, Hospital Universitari Germans Trias i Pujol, Badalona, Spain), and J. María Pina (Programa de Tuberculosi Regió Centre, Terrassa, Spain), for technical assistance and helpful discussions. The authors are also indebted to C. Rodrigo (Servei de Pediatria, Hospital Universitari Germans Trias i Pujol, Badalona), N. Díez and A. Escribano (both Servicio de Pediatría, Hospital Clínico Universitario, Valencia, Spain) for their kind help in enrolling children for the control groups.

\section{REFERENCES}

1 Migliori GB, Hopewell PC, Blasi F, et al. Improving the TB case management: The International Standards for Tuberculosis Care Eur Respir J 2006; 28: 687-690.

2 Lalvani A, Millington KA. T-cell-based diagnosis of childhood tuberculosis infection. Curr Opin Infect Dis 2007; 20: 264-271.

3 Jasmer RM, Nahid P, Hopewell PC. Clinical practice. Latent tuberculosis infection. N Engl J Med 2002; 347: 1860-1866.

4 Andersen P, Munk ME, Pollock JM, et al. Specific immune-based diagnosis of tuberculosis. Lancet 2000; 356: 1099-1104.

5 Brock I, Weldingh K, Leyten EM, et al. Specific T-cell epitopes for immunoassay-based diagnosis of Mycobacterium tuberculosis infection. J Clin Microbiol 2004; 42: 2379-2387.

6 Connell TG, Curtis N, Ranganathan SC, et al. Performance of a whole blood interferon gamma assay for detecting latent infection with Mycobacterium tuberculosis in children. Thorax 2006; 61: 616-620.

7 Dominguez J, Ruiz-Manzano J, De Souza-Galvao M, et al. Comparison of two commercially available gamma interferon blood tests for immunodiagnosis of tuberculosis. Clin Vaccine Immunol 2008; 15: 168-171.

8 Ewer K, Deeks J, Alvarez L, et al. Comparison of T-cell-based assay with tuberculin skin test for diagnosis of Mycobacterium tuberculosis infection in a school tuberculosis outbreak. Lancet 2003; 361: 1168-1173.

9 Ferrara G, Losi M, D'Amico R, et al. Use in routine clinical practice of two commercial blood tests for diagnosis of infection with Mycobacterium tuberculosis: a prospective study. Lancet 2006; 367: 1328-1334.

10 Goletti D, Stefania C, Butera O, et al. Accuracy of immunodiagnostic tests for active tuberculosis using single and combined results: a multicenter TBNET-Study. PLOS ONE 2008; 3: e3417.

11 Domínguez J, De Souza-Galvao M, Ruiz-Manzano J, et al. T-cell responses to the Mycobacterium tuberculosis-specific antigens in active 
tuberculosis patients at the beginning, during, and after antituberculosis treatment. Diagn Microbiol Infect Dis 2009; 63: 43-51.

12 Menzies D, Pai M, Comstock G. Meta-analysis: new tests for the diagnosis of latent tuberculosis infection: areas of uncertainty and recommendations for research. Ann Intern Med 2007; 146: 340-354.

13 Ruiz-Manzano J, Blanquer R, Calpe JL, et al. SEPAR Guidelines. Diagnostic and treatment of tuberculosis. Arch Bronconeumol 2008; 44: 551-566.

14 Connell TG, Ritz N, Paxton GA, et al. A three-way comparison of tuberculin skin testing, QuantiFERON-TB gold and T-SPOT.TB in children. PLOS ONE 2008; 3: e2624.

15 von Reyn CF, Horsburgh CR, Olivier KN, et al. Skin test reactions to Mycobacterium tuberculosis purified protein derivative and Mycobacterium avium sensitin among health care workers and medical students in the United States. Int J Tuberc Lung Dis 2001; 5: $1122-1128$.
16 Alcaide Megias J, Altet Gomez MN, Canela i Soler J. [Epidemiology of tuberculosis.] An Esp Pediatr 2000; 53: 449-457.

17 Bierrenbach AL, Floyd S, Cunha SC, et al. A comparison of dual skin test with mycobacterial antigens and tuberculin skin test alone in estimating prevalence of Mycobacterium tuberculosis infection from population surveys. Int J Tuberc Lung Dis 2003; 7: 312-319.

18 Fairchok MP, Rouse JH, Morris SL. Age-dependent humoral responses of children to mycobacterial antigens. Clin Diagn Lab Immunol 1995; 2: 443-447.

19 Larsson LO, Skoogh BE, Bentzon MW, et al. Sensitivity to sensitins and tuberculin in Swedish children. II. A study of preschool children. Tubercle 1991; 72: 37-42.

20 Leyten EM, Arend SM, Prins C, et al. Discrepancy between Mycobacterium tuberculosis-specific gamma interferon release assays using short and prolonged in vitro incubation. Clin Vaccine Immunol 2007; 14: 880-885. 